\title{
THERE'S NO FORCING A LEAST UPPER BOUND
}

\author{
ROBERT S. LUBARSKY
}

(Communicated by Thomas J. Jech)

\begin{abstract}
While it is possible to have an increasing $\omega$-sequence of $c$-degrees with a least upper bound in a particular model of $\mathrm{ZF}$, we show that it is not possible to force a sequence with a least upper bound absolute among all generic extensions of the original model
\end{abstract}

In [H], Hájek forces over $L$ a sequence of reals $\left\langle a_{n} \mid n \in \omega\right\rangle$ which, when coded in some trivial manner into a real $\alpha$, is a good candidate for the least upper bound of the $c$-degrees of the $a_{n}$ 's. "Good candidate" means that any upper bound which does not collapse $\aleph_{1}$ also constructs the sequence. He left open the question of whether it is actually the least upper bound. We show here that it is not, which means that there is no least upper bound.

Hajek's partial order is the $\omega$-step iteration (with finite support) of the Jensen-Johnsbraten forcing [JJ]. The absoluteness comes about because any pair of JJ-generics (over the same ground model satisfying $V=L$ ) collapses $\aleph_{1}$. Also, a JJ-generic $G$ over $L$ is definable in $L[G]$. So if $\kappa_{1}^{V}=\aleph_{1}^{L}$ then the sequence of generics is definable as the $\omega$-sequence $\left\langle a_{0}, a_{1}, \ldots\right\rangle$ such that $a_{n+1}$ is the unique set satisfying the appropriate definition over $L\left[a_{n}\right]$.

The reason that Hájek's sequence is not necessarily definable if $\kappa_{1}^{L}$ is collapsed is that for each $n$ there may be many such generics with no way of distinguishing $a_{n+1}$ from the rest. More particularly, there may be a perfect tree the $n$th level of which is a set of mutually generic reals for the $n$th partial order. Even if Hájek's generic is a path through this tree, there is no guarantee that it is constructible from the tree.

The theorem below is a general statement which implies as a particular instance that Hájek's sequence $\left\langle a_{0}, a_{1}, \ldots\right\rangle$ has no l.u.b. We chose this presentation in part because it focuses attention on the relevant points, which do not include special features of a partial order. This result is actually just another application of the main idea of $[\mathrm{L}]$ of hiding a generic as a path through a tree. There, though, it was used in somewhat the opposite direction, showing that a particularly recalcitrant set of hyperdegrees does have a minimal upper bound.

Received by the editors April 19, 1987.

1980 Mathematics Subject Classification (1985 Revision). Primary 03E40; Secondary 03E45.

Key words and phrases. Degree of constructibility, forcing. 
Definition 1. For $\mathscr{M} \vDash \mathrm{ZF}$ standard, $A \leq{ }_{\mathscr{M}} B$ if $A \in \mathscr{M}[T C(B)]$. The equivalence classes of $\leq_{\mathscr{K}}$ are the $\mathscr{M}$-degrees. By convention, we restrict our attention to standard sets $A$ such that $\mathscr{M}[T C(A)] \vDash \mathrm{ZF}$.

Theorem 2. Let $\mathscr{M}$ be a countable standard model of $Z F, \mathscr{P}_{\omega}=\lim _{n \in \omega} \mathscr{P}_{n} \in$ $\mathscr{M}$ a limit of complete Boolean algebras, and $\left\langle G_{0}, G_{1}, \ldots\right\rangle$ a $\mathscr{P}_{\omega}$-generic over $\mathscr{M}$, with $G_{n} \mathscr{P}_{n}$-generic and $G_{n+1} \Varangle_{\mathscr{M}} G_{n}$. Then $\left\{\mathscr{M}-\operatorname{deg}\left(G_{n}\right) \mid n \in \omega\right\}$ has no least upper bound in the $\mathscr{M}$-degrees.

Remarks. In the statement of the theorem, the kind of limit taken is left ambiguous, since it does not matter. Note that $\mathscr{P}_{\omega} \neq \mathscr{P}_{0} * \mathscr{P}_{1} * \cdots$. Rather $\mathscr{P}_{\omega}=\mathscr{Q}_{0} * \mathscr{Q}_{1} * \cdots$ and $\mathscr{P}_{n}=\mathscr{Q}_{0} * \cdots * \mathscr{Q}_{n}$ for an appropriate choice of $\mathscr{Q}_{i}$ 's. We freely identify $\mathscr{P}_{n}$ with its canonical image in $\mathscr{P}_{\omega}$ or $\mathscr{P}_{m}(m>n)$. We assume that $1 \Vdash \mathscr{Q}_{n}$ is a complete atomless Boolean algebra", by working beneath a condition forcing such. Our final forcing partial order $\mathscr{P}$ is an $\omega$ iteration, but is neither a direct nor an inverse limit. For more details on forcing and limits in the language of Boolean algebra, see [J].

The idea of the proof is to take the sequence $\left\langle G_{0}, G_{1}, \ldots\right\rangle$ and hide it in a bigger model. This is done by including many $\mathscr{Q}_{n+1}$-generics over $\mathscr{M}\left[G_{n}\right]$, to keep $G_{n+1}$ from being a canonical choice, even with parameter $G_{n}$. Thus we want a tree of order type $\omega^{<\omega}$, and at node $\sigma$ a generic over $\mathscr{P}_{|\sigma|}$ which (except for $\sigma=\varnothing$ ) extends the generic for $\sigma|| \sigma \mid-1$, such that $\left\langle G_{0}, G_{1}, \ldots\right\rangle$ is a "generic" path through the tree. Thus the tree itself is an upper bound for $\left\{\mathscr{M}-\operatorname{deg}\left(G_{n}\right) \mid n \in \omega\right\}$, since each $G_{n}$ is a node on the tree. Furthermore, the tree does not construct a previously given upper bound $G_{\omega}$ by the "genericity" of the path $\left\langle G_{0}, G_{1}, \ldots\right\rangle$.

Such a tree can be constructed generically, using the partial order $\mathscr{P}$ with conditions $p$ of partial functions having domain $\omega^{<\omega}$ and $p(\sigma) \in \mathscr{P}_{|\sigma|}$. There are conflicting demands upon $\mathscr{P}$, however. $\mathscr{P}$ must include all conditions in $\mathscr{P}_{\omega}$, which might necessitate conditions with infinite support. It must also have some sort of finite support, in order to allow for the appropriate diagonalization. (Such diagonalization is the "genericity" of $\left\langle G_{0}, G_{1}, \ldots\right\rangle$ as a path through the $\mathscr{P}$-generic, as referred to above.) Thus a condition $p$ will have as support finitely many paths through $\omega^{<\omega}$.

Definition 3. Working in $\mathscr{M}$, let $\mathscr{P}$ be $\left\{p: \omega^{<\omega} \rightarrow \bigcup_{n \in \omega} \mathscr{P}_{n} \mid p\right.$ is partial and for some $f_{1}, \ldots, f_{n} \in \omega^{\omega}, n$ finite

(a) $\operatorname{dom} p=\left\{f_{i} \uparrow j \mid i \leq n, j<\omega\right\}$

(b) $p(\sigma) \in \mathscr{P}_{|\sigma|}$

(c) $\pi_{j}^{j+1}\left(p\left(f_{i} \nmid j+1\right)\right)=p\left(f_{i} \uparrow j\right)$ and

(d) in $\mathscr{P}_{\omega}, \lim _{j \rightarrow \omega} p\left(f_{i} \mid j\right) \neq \mathscr{\mathscr { C }}_{(i)} 0 . \quad\left(\pi_{l}^{k}\right.$ is the canonical projection from $\mathscr{P}_{k}$ to $\mathscr{P}_{1}$.)

Let $q \leq p$ if $\operatorname{dom}(q) \supseteq \operatorname{dom}(p)$, and $q(\sigma) \leq_{\mathscr{P}_{|\sigma|}} p(\sigma)$ for $\sigma \in \operatorname{dom} p$.

Let $G_{\omega}$ be an upper bound of the $G_{n}$ 's in the $\mathscr{M}$-degrees. We want a $\mathscr{P}$ generic $G$ such that: 
(a) $\forall n \exists \sigma G_{n}=\{p(\sigma) \mid p \in G\}$ and

(b) $G_{\omega} \notin \mathscr{M}[T C(G)]$.

Notice that such a $G$ is an upper bound for $\left\{\mathscr{M}-\operatorname{deg}\left(G_{n}\right) \mid n \in \omega\right\}$ by (a), which does not construct an alleged l.u.b. $G_{\omega}$, thus proving the theorem.

The following definitions and lemma are useful, and quite natural.

Definition 4. For $\sigma \in \omega^{<\omega}$, let $G(\sigma)=\{p(\sigma) \mid p \in G\}$. (Thus condition (a) above is " $\forall n \exists \sigma G_{n}=G(\sigma)$ ".)

We want to take the Boolean meet of conditions, even if they come from different Boolean algebras. Hence,

Definition 5. For $\sigma, \tau \in \omega^{\leq \omega}$, let $m(\sigma, \tau)=\sup \{m|\sigma| m=\tau \mid m\}$, the length of agreement of $\sigma$ and $\tau$.

For $p \in \mathscr{P}, r \in \mathscr{P}_{n}, \sigma \in \omega^{n}$, let $p \wedge_{\sigma} r \in \mathscr{P}$ have domain $\operatorname{dom} p \cup$ $\{\sigma|m| m \leq|\sigma|\}$, and have $\left(p \wedge_{\sigma} r\right)(\tau)=p(\tau) \wedge \pi_{m(\sigma, \tau)}^{n}(r)$. Notice that $p \wedge_{\sigma} r$ is undefined if $p(\sigma) \perp r$, by clause (d) in the definition of $\mathscr{P}$.

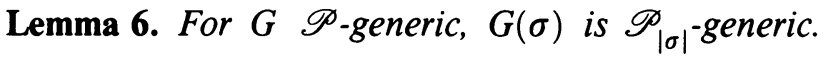

Proof. Suppose $D \subseteq \mathscr{P}_{|\sigma|}$ in $\mathscr{M}$ is dense. Given $p \in \mathscr{P}$, let $r \leq p(\sigma), r \in D$. $p \wedge_{\sigma} r$ as defined above is a condition, because if

$$
\lim _{j \rightarrow \omega}\left(\left(p \wedge_{\sigma} r\right)\left(f_{i} \uparrow j\right)\right)=\pi_{m\left(f_{i}, \sigma\right)}^{|\sigma|}(r) \wedge \lim _{j \rightarrow \omega} p\left(f_{i} \uparrow j\right)=0
$$

then $\lim _{j \rightarrow \omega} p\left(f_{i} \mid j\right) \leq \neg\left[\pi_{m\left(f_{i}, \sigma\right)}^{|\sigma|}(r)\right]$. But then

$$
p\left(f_{i} \uparrow m\left(f_{i}, \sigma\right)\right) \leq \neg\left[\pi_{m\left(f_{i}, \sigma\right)}^{|\sigma|}(r)\right],
$$

which contradicts $r \leq p(\sigma)$. So $p \wedge_{\sigma} r \leq p$ and $\left(p \wedge_{\sigma} r\right)(\sigma) \in D$, which means that generically $G(\sigma)$ will hit each dense $D \subseteq \mathscr{P}_{|\sigma|}$ from $\mathscr{M}$.

Now we construct $G \mathscr{P}$-generic over $\mathscr{M}$, satisfying (a) and (b). The construction takes $\omega$-many stages. The first step at stage $n$ is to hit the $n$th dense set, and in such a way that $G_{n}$ remains compatible with (the current state of) $G\left(\sigma_{n}\right)$ (some $\sigma_{n}$ suitably chosen). The second step is the diagonalization, to ensure that the $n$th term, when finally evaluated in $M[G]$, does not equal $G_{\omega}$ (again respecting $G_{n}$ ).

Let $\left\langle D_{n} \mid n \in \omega\right\rangle,\left\langle t m_{n} \mid n \in \omega\right\rangle$ list, respectively, the dense subsets of $\mathscr{P}$ in $\mathscr{M}$ and the terms for members of $\mathscr{M}[G]$ in $\mathscr{M}$.

Start with $\sigma_{0}=\varnothing$ and $p_{0}=\left\langle\phi, 1_{\mathscr{O}_{0}}\right\rangle$. Assume inductively that at stage $n$ we have $p_{n} \in \mathscr{P}$ and $\sigma_{n} \in \operatorname{dom} p_{n}$ of length $n$ so that $p_{n}\left(\sigma_{n}\right) \in G_{n}$.

The following lemma will be used several times. It justifies extending a condition $p \in \mathscr{P}$ to satisfy a density requirement while retaining the compatability of $G(\sigma)$ and $G_{|\sigma|}$ for some given $\sigma$.

Lemma 7. Let $p \in \mathscr{P}, \sigma \in \operatorname{dom} p$, and $D \subseteq \mathscr{P}$ dense and in $\mathscr{M}$. Then $\left\{p^{\sigma} \in \mathscr{P} \mid \exists q \in D q \leq p\right.$ and $\left.q(\sigma)=p^{\sigma}\right\}$ is dense beneath $p(\sigma)$ in $\mathscr{P}_{|\sigma|}$.

Proof of Lemma. Let $r \leq_{\mathscr{S}_{|\sigma|}} p(\sigma)$. Extend $p \wedge_{\sigma} r$ to $q \in D . q(\sigma) \leq r$ is as desired. 
To complete the first step of stage $n$, apply Lemma 7 with $p=p_{n}, \sigma=\sigma_{n}$, and $D=D_{n}$. By genericity of $G_{n}$, let $p_{n}^{\prime} \leq p_{n}, p_{n}^{\prime} \in D_{n}, p_{n}^{\prime}\left(\sigma_{n}\right) \in G_{n}$.

Now apply Lemma 7 with $p=p_{n}^{\prime}, \sigma=\sigma_{n}$, and $D=\left\{p \mid p \|\right.$ " $t m_{n} \in$ $\mathscr{M}\left[G\left(\sigma_{n}\right)\right]$ " $\}$, to get $p_{n}^{\prime \prime} \leq p_{n}^{\prime}$ deciding “ $t m_{n} \in \mathscr{M}\left[G\left(\sigma_{n}\right)\right]$ ", $p_{n}^{\prime \prime}\left(\sigma_{n}\right) \in G_{n}$. If $p_{n}^{\prime \prime} \vDash$ " $t m_{n} \in \mathscr{M}\left[G\left(\sigma_{n}\right)\right]$ ”, then let $p_{n}^{\prime \prime \prime}$ be $p_{n}^{\prime \prime}$. If $p_{n}^{\prime \prime} \vDash$ “t的 $\notin \notin \mathscr{M}\left[G\left(\sigma_{n}\right)\right]$ ", then we want to apply Lemma 7 with $p=p_{n}^{\prime \prime}, \sigma=\sigma_{n}$, and $D=D_{t m_{n}}=_{\text {def }}$ $\left\{q \mid q \perp p_{n}^{\prime \prime}\right.$ or there are $q_{1}, q_{2} \leq q q_{1}\left(\sigma_{n}\right)=q_{2}\left(\sigma_{n}\right)=q\left(\sigma_{n}\right)$ and $q_{1}, q_{2}$ are t $m_{n}$-incompatible (see below) $\}$.

Lemma 8. $D_{t m_{n}}$ is dense.

Remark. " $t m_{n}$-incompatible" means "forces contradictory statements about $t m_{n} ": q_{1} \Vdash \varphi$ and $q_{2} \Vdash \neg \varphi$, for some $\varphi$ all of whose terms are canonical names for members of $\mathscr{M}$ or $t m_{n}$ itself.

Proof. Let $\tilde{q} \leq p_{n}^{\prime \prime}$. Let $G$ be $\mathscr{P}$-generic. $G\left(\sigma_{n}\right)$ is $\mathscr{P}_{n}$-generic by Lemma 6, so $\mathscr{P} \simeq \mathscr{P}_{n} * \mathscr{P} / G\left(\sigma_{n}\right)$. Furtheremore, by hypothesis on $p_{n}^{\prime \prime}, \operatorname{tm}_{n}^{\mathscr{M}[G]} \notin$ $\mathscr{M}\left[G\left(\sigma_{n}\right)\right]$. So there are $t m_{n}$-incompatible $q_{1}^{\prime}, q_{2}^{\prime}$ in $\mathscr{P} / G\left(\sigma_{n}\right)$, since otherwise $t m_{n}^{\mathscr{M}[G]}$ would be definable in $\mathscr{M}\left[G\left(\sigma_{n}\right)\right]$ as $\left\{x \mid\right.$ for some $r \in \mathscr{P} / G\left(\sigma_{n}\right), r \Vdash$ “ $\check{x} \in t m_{n}$ " $\}$. Let $q^{\prime} \in G\left(\sigma_{n}\right)$ force such about $q_{1}^{\prime}$ and $q_{2}^{\prime}$. Then $q=\tilde{q} \wedge_{\sigma_{n}} q^{\prime}$ is as desired, with witnesses $q_{1}=\left\langle q\left(\sigma_{n}\right), q \wedge q_{1}^{\prime}\right\rangle$ and $q_{2}=\left\langle q\left(\sigma_{n}\right), q \wedge q_{2}^{\prime}\right\rangle$.

By genericity of $G_{n}$, let $p_{n}^{\prime \prime 1 / 2} \leq p_{n}^{\prime}$ be such that $p_{n}^{\prime \prime 1 / 2}\left(\sigma_{n}\right) \in G_{n}$, and $p_{n}^{\prime \prime 1 / 2} \in$ $D_{t m_{n}}$ with witnesses $q_{1}$ and $q_{2}$. Let $p_{n}^{\prime \prime \prime}$ be a $q_{i}$ which forces something about $t m_{n}$ false for $G_{\omega}$. That is, for $\varphi$ the witness to the $t m_{n}$-incompatability of $q_{1}$ and $q_{2}$, and $\psi=\varphi$ or $\neg \varphi, p_{n}^{\prime \prime \prime}$ is the $q_{i}$ such that $q_{i} \Vdash \psi$ and $M\left[G_{\omega}\right] \vDash \neg \psi$ (with $t m_{n}$ interpreted as $G_{\omega}$ in the latter context).

Choose an $m$ so that $\sigma_{n}^{\wedge} m \notin \operatorname{dom} p_{n}^{\prime \prime \prime}$. Let $\sigma_{n+1}=\sigma_{n}^{\wedge} m$ and $p_{n+1}=$ $p_{n}^{\prime \prime \prime} \cup\left\{\left\langle\sigma_{n+1} \wedge 0^{t}, p^{\prime \prime \prime}\left(\sigma_{n}\right)\right\rangle \mid t \in \omega\right\}$. This completes the induction.

Let $G=\left\{p \mid p \geq p_{n}\right.$ for some $\left.n\right\}$. Notice that for $m>n, \sigma_{m}$ extends $\sigma_{n}$, so $p_{m}\left(\sigma_{m}\right) \leq G_{m}$ implies $p_{m}\left(\sigma_{n}\right) \in G_{n}$. Consequently, by the first step in the construction, $G\left(\sigma_{n}\right) \subseteq G_{n}$. Each is $\mathscr{P}_{n}$-generic. Therefore $G\left(\sigma_{n}\right)=G_{n}$. So $G$ is an upper bound of the $G_{n}$ 's. By the diagonalization of the second step, $G_{\omega} \notin \mathscr{M}[G]$. (Note that in the first case of the second step, $t m_{n}^{\mathscr{K}[G]} \in$ $\mathscr{M}\left[G\left(\sigma_{n}\right)\right]=\mathscr{M}\left[G_{n}\right]$, and $G_{\omega} \notin \mathscr{M}\left[G\left(\sigma_{n}\right)\right]$ for any $n$ since $G_{n+1} \notin \mathscr{M}\left[G_{n}\right]$ by hypothesis.) Therefore $G_{\omega}$ is not the least upper bound of the $G_{n}$ 's.

Question. $0^{\# / 0}=$ l.u.b. $0^{\#^{n}}$, so least upper bounds are possible. Is there a countable standard model of ZF with an absolute least upper bound (that is, a degree which is an l.u.b. in all models with the same ordinals) that does not collapse cardinals? 


\section{REFERENCES}

[H] P. Hájek, Some results on degrees of constructibility, Higher Set Theory, Proceedings, Oberwolfach, Germany, April 13-23, 1977, (G. H. Müller and D. S. Scott, eds.) Lecture Notes in Math., vol. 669, Springer-Verlag, Berlin and New York, 1978, pp. 55-71.

[J] T. Jech, Set theory, Academic Press, New York, 1978.

[JJ] R. Jensen and H. Johnsbraten, A new construction of a non-constructible $\Delta_{3}^{1}$ subset of $\omega$, Fund. Math. 81 (1974), 279-290.

[L] R. Lubarsky, Sacks forcing sometimes needs help to produce a minimal upper bound, J. Symbolic Logic 54 (1989) (to appear).

Department of Mathematics, Cornell University, Ithaca, New York 14853

Current address: Franklin \& Marshall College, Department of Mathematics and Astronomy, P.O. Box 3003, Lancaster, Pennsylvania 17604-3003 